\title{
CONCEITO de GRUPO NA PERCEPÇÃO dE ENFERMEIROS NA ÁREA DE SAÚdE MENTAL E PSIQUIATRIA ${ }^{1}$
}

\author{
Luciene Simões Spadini \\ Maria Conceição Bernardo de Mello e Souza ${ }^{3}$
}

O estudo teve como objetivo identificar, entre enfermeiros da rede de Saúde Mental de um município do interior paulista, a compreensão que têm sobre grupo. Trata-se de estudo qualitativo do tipo descritivo-exploratório. Os sujeitos foram 26 enfermeiros da rede. Os dados foram coletados por meio de entrevista individual. Para análise, utilizou-se a análise temática. Alguns enfermeiros definem grupo como uma modalidade terapêutica; outros relacionam com reuniões multiprofissionais e outros, ainda, relacionam o conceito com coordenação dos grupos. O tema carece de melhor enfoque, tanto na assistência como no ensino, para que haja aplicação apropriada desse recurso como fonte das ações de enfermagem.

Descritores: Enfermagem; Saúde Mental; Enfermagem Psiquiátrica.

\footnotetext{
${ }^{1}$ Artigo extraído da dissertação de Mestrado "A inserção do enfermeiro no contexto de saúde mental: o trabalho com grupos" apresentado à Escola de Enfermagem de Ribeirão Preto, Universidade de São Paulo, Centro Colaborador da OMS para o Desenvolvimento da Pesquisa em Enfermagem, SP, Brasil.

${ }^{2}$ Enfermeira, Mestre em Psiquiatria, Hospital das Clínicas, Faculdade de Medicina de Ribeirão Preto, Universidade de São Paulo, SP, Brasil. E-mail: luspadini@hotmail.com.

${ }^{3}$ Enfermeira, Livre Docente, Professor Associado, Escola de Enfermagem de Ribeirão Preto, Universidade de São Paulo, Centro Colaborador da OMS para o Desenvolvimento da Pesquisa em Enfermagem, SP, Brasil. E-mail: consouza@eerp.usp.br.
} 


\section{THE GROUP CONCEPT ACCORDING TO MENTAL HEALTH AND PSYCHIATRY NURSES}

This study aimed to identify, among nurses in the Mental Health network in a city in the interior of São Paulo State, their understanding about the "group" theme. A qualitative, descriptive and exploratory study was developed. Twenty-six nurses participated. The data were collected by means of individual interviews. For data analysis, thematic analysis was used. Some of the nurses define group as a therapeutic modality; others relate it with multi-professional meetings; and still others even relate the concept with group coordination. This theme lacks a better approach, both in care and teaching, with a view to the appropriate application of this resource as a source of nursing actions.

Descriptors: Nursing; Mental Health; Psychiatric Nursing.

\section{CONCEPTO DE GRUPO EN LA PERCEPCIÓN DE ENFERMEROS EN EL ÁREA DE Salud Mental y Psiquiatría}

La finalidad del estudio fue identificar entre los enfermeros de la red de Salud Mental de un municipio del interior del estado de São Paulo la comprensión que tienen sobre grupo. Estudio cualitativo del tipo descriptivo-exploratorio. Fueron 26 enfermeros los participantes de esa investigación. Los datos fueron recolectados a través de entrevista individual. Para análisis de los datos, se utilizó el análisis temático. Algunos participantes definen grupo como una modalidad terapéutica; otros lo relacionan con reuniones multiprofesionales, y además hay otros que relacionan grupo con la coordinación de los mismos. El tema carece de un mejor enfoque, tanto en la atención como en la enseñanza, con vistas a la aplicación apropiada de ese recurso como fuente de las acciones de enfermería.

Descriptores: Enfermería; Salud Mental; Enfermería Psiquiátrica.

\section{Introdução}

O termo grupo é recente e possui um vocábulo similar em vários idiomas, com origens diversas. Segundo os linguistas, o termo italiano groppo origina-se do alemão kruppa, significando masa redondeada; enquanto o termo francês groupe vem do italiano groppo ou gruppo, como termo técnico de belas-artes, indicando vários indivíduos pintados ou esculpidos que compõem a ideia de círculo, vindo a designar uma reunião de pessoas ${ }^{(1)}$.

Um conjunto de pessoas constitui um grupo, um conjunto de grupos constitui uma comunidade e um conjunto de comunidades que interagem representa uma sociedade. O indivíduo passa a maior parte de sua vida convivendo e interagindo em grupos, tornando-se importante o conhecimento e a utilização da estratégia grupal para o estudo das relações humanas ${ }^{(2)}$.
É vaga e imprecisa a definição de grupo, pois pode ser o conjunto de duas ou três pessoas, como também uma família, uma gangue, uma classe ou um grupo terapêutico ${ }^{(3)}$.

Grupo, porém, não é um somatório de pessoas, mas uma entidade com mecanismos específicos, próprios e com leis, e todos os integrantes estão unidos para o alcance de um objetivo comum ${ }^{(1)}$.

Existem dois tipos de grupos, o primário e o secundário. A família seria um exemplo de grupo primário e a escola, o trabalho, as instituições, exemplos de grupos secundários. Nos grupos, cada ser encontra um lugar, um papel que, por sua vez, constitui a maneira de ser do gênero humano e, nesse espaço, desempenham-se os papéis, segundo a história de cada um ${ }^{(4)}$. 
A principal diferença entre os grupos ocorre na sua finalidade, isto é, para que eles foram criados e compostos. Os grupos podem ser divididos em dois ramos genéricos, os operativos e os terapêuticos. Os operativos podem ter várias aplicações, inclusive a terapêutica, mesmo que seja utilizada exclusivamente a abordagem psicanalítica. Os grupos operativos envolvem os seguintes campos: ensino/aprendizagem, institucionais (empresas, igreja, associações, escolas, exército etc.) e comunitários programas de saúde mental ${ }^{(3,5)}$.

Os grupos psicoterápicos são aqueles de ação exclusivamente psicoterápica, isto é, aqueles que possibilitam aos integrantes aquisição de insight dos aspectos inconcientes de si mesmos e do grupo. Existem várias abordagens para se trabalhar em grupos com finalidade exclusivamente terapêutica como, por exemplo, a psicodramática, a psicanalítica, a da teoria sistêmica, cognitivo-comportamental e a abordagem múltipla.

Em relação às modalidades de grupo que podem ser utilizadas pela enfermagem, encontram-se o grupo operativo, que tem seu objetivo centrado em uma tarefa que pode ser o aprendizado, as dificuldades, a cura, o diagnóstico e outros ${ }^{(6)}$.

No campo do ensino/aprendizagem, o grupo operativo como técnica para trabalhar com pequenos grupos de estudantes tem sido utilizada no sentido do referencial pichoniano, que abre a possibilidade desse constante repensar e, fundamentalmente, de refazer a prática do ensinar, incorporando estudantes e professores como sujeitos críticos e criativos que possam transformar o cotidiano ${ }^{(7)}$.

Quanto aos grupos de autoajuda, é uma modalidade do grupo operativo terapêutico e consistem em grupos de formação espontânea de pessoas que se sentem identificadas por características semelhantes entre si. A utilização terapêutica do grupo de autoajuda merece destaque, tanto pela sua eficácia como pelo largo âmbito de sua aplicação e expansão(2). Os grupos de autoajuda são organizados ao redor de uma experiência comum, podendo ou não receber consultoria de um provedor da saúde, como um enfermeiro, porém, eles são operados pelos seus membros $^{(8)}$.

A modalidade grupo de sala de espera, geralmente, é utilizada para preencher o tempo ocioso das pessoas que esperam por atendimento ou pelo familiar que está sendo assistido nos serviços de saúde. Nas instituições que oferecem atendimento psicológico gratuito, vem ocorrendo procura cada vez maior pelo serviço. Assim, fica evidente a necessidade de diversificação e busca de novas soluções para essa clientela.

O principal objetivo dos grupos de apoio é ajudar seus membros a enfrentar o estresse da vida, sendo que o foco desse tipo de grupo está nos pensamentos, sentimentos e comportamentos disfuncionais para os quais são oferecidos apoio emocional e informações críticas para o aumento das capacidades de seus membros, para o enfrentamento e a solução dos problemas, reforçando o sistema de apoio entre os pacientes ${ }^{(8)}$.

Os grupos nos serviços de semi-internação, ambulatórios e no contexto hospitalar são conduzidos e organizados dependendo de diversas situações.

Estudo realizado em um hospital dia aponta que os grupos comunitários são norteados por princípios de comunidade terapêutica, reconhecidos como espaço de reflexão sobre o tratamento oferecido. Os seus conteúdos são voltados para a doença e suas repercussões psicossociais. Os mesmos autores descrevem como o grupo é percebido pelos participantes, repercutindo como espaço integrador e favorecedor para o surgimento do novo ${ }^{(9)}$.

O atendimento em ambulatórios, nos serviços de saúde mental, oferece como uma das modalidades as terapias grupais. O recurso é justificado pela economia de tempo, exigência de produtividade, necessidade de diminuir filas, sendo preciso, todavia, avaliar a adequação em relação à indicação dessa modalidade ao usuário. Dados de pesquisa apontam que as propostas de atendimento psicoterápico em ambulatórios de saúde mental têm sido pouco eficientes, resultando em altos índices de abandono, reinternação e cronificação ${ }^{(10-11)}$.

Dependendo da forma com que é conduzido e organizado, o recurso grupal pode ser usado em diversas situações e para diversos fins, melhorando muito a qualidade de vida das pessoas nos seus relacionamentos, na família, no trabalho e na sociedade, como um todo ${ }^{(12)}$.

Tendo em vista o apontado anteriormente, este estudo buscou identificar, entre os enfermeiros da rede de Saúde Mental e Psiquiatria de um município do interior paulista, a compreensão que têm sobre a temática grupo.

\section{Metodologia}

Trata-se de estudo de natureza qualitativa do tipo descritivo-exploratório. O tema deste estudo, Conceito de grupo na percepção de enfermeiros na área de Saúde Mental e Psiquiatria, foi extraído da dissertação de mestrado intitulada A inserção do enfermeiro no contexto de Saúde Mental: o trabalho com grupos. Os dados foram obtidos por meio de entrevistas individuais somente após a permissão dos enfermeiros, mediante as condições da Resolução nº196/96, do Conselho Nacional de Saúde, sobre pesquisa envolvendo seres humanos ${ }^{(13)}$ e, após apreciação do Comitê de Ética em Pesquisa do Hospital das Clínicas da Faculdade de Medicina de Ribeirão Preto Universidade de São Paulo, e assinatura pelos participantes do termo de consentimento informado livre e esclarecido, bem como a autorização dos locais de estudo. Para a coleta de dados e realização das entrevistas, foi utilizado roteiro previamente elaborado com algumas questões norteadoras, e uma delas foi: o que é grupo para você?

As entrevistas foram realizadas em um único encontro, em sala reservada nas instituições, com data e horário pré-estabelecidos. Duraram, em média, 15 minutos, foram gravadas e posteriormente transcritas na íntegra pelo próprio pesquisador.

A entrevista é o procedimento utilizado em pesquisa de campo na qual o pesquisador busca obter informações contidas nas falas dos atores sociais. Ela se insere como 
meio de coleta de dados de fatores correlatos que vivenciam a realidade que está sendo investigada ${ }^{(14)}$.

Os participantes desta investigação foram 26 enfermeiros da rede de serviços de Psiquiatria e Saúde Mental de uma cidade do interior paulista e se constituiu por serviços abertos, semiabertos e de internação integral.

Após a coleta de dados e transcrição das entrevistas, o material foi organizado, lido quantas vezes foram necessárias, sendo realizada a identificação de temas, provenientes dos depoimentos. Após isso, foram analisados conforme os objetivos desta investigação.

A análise do material coletado, na pesquisa qualitativa, deve ser feita com três finalidades distintas: identificar e conhecer as informações, confirmar ou refutar os pressupostos da pesquisa e, em um terceiro momento, ampliar o conhecimento sobre o tema estudado ${ }^{(14)}$.

Classificar significa organizar ou ordenar em uma série de diferentes dados de classes o todo ou o universo estudado, dividido em partes, agrupando os dados em categorias para serem analisados ${ }^{(15)}$.

A análise e a discussão dos dados foram embasadas nos passos propostos por Minayo ${ }^{(14)}$ :

1- ordenação dos dados - reunir todo o material, ou seja, organizar os dados (início da classificação);

2- classificação dos dados - leituras exaustivas do conteúdo transcrito das entrevistas; apreensão das ideias centrais e estabelecimento das categorias empíricas do estudo;

3- análise final - articular o material teórico e o empírico da pesquisa, orientada pelos objetivos da investigação.

Para a elaboração dos temas, foram feitas leituras exaustivas dos dados coletados e uma análise das temáticas que emergiram nas entrevistas, relacionados à revisão da literatura e aos objetivos da pesquisa. No atual estudo, realizou-se um recorte, enfocando o tema "conceito de grupo".

\section{Resultados e Discussões}

Do total de 44 enfermeiros admitidos nos serviços, $26(59 \%)$ aceitaram participar da pesquisa. Todos os entrevistados participam ou já participaram de atividades grupais em seu serviço. Dos sujeitos envolvidos, 15 $(57,7 \%)$ estão no quadro de profissionais das unidades de internação integral, nove (31\%) nos serviços abertos e dois (7,7\%) nos serviços semiabertos.

Dos 26 enfermeiros, 24 são do sexo feminino $(92,4 \%)$ e dois do sexo masculino (7,6\%). A idade entre os mesmos variou de 24 a 52 anos. Analisando a instituição formadora, 15 (57,7\%) concluíram o curso de graduação em enfermagem em escola pública e 11 (42,3\%) em escolas privadas. Quanto ao ano de formação, variou de 1979 a 2003.

Em relação à atuação dos enfermeiros nos grupos, seis (23\%) referiram ser coordenadores de grupo;,seis $(23 \%)$ coterapeutas, dois (7,7\%) observadores e $12(46,3 \%)$ informaram ser participantes dos grupos realizados nos serviços de psiquiatria e saúde mental.

Foi analisado e discutido como os enfermeiros têm compreendido e conceituado "grupo".
Observaram-se, nos resultados obtidos, várias concepções de grupo apontadas pelos enfermeiros dos serviços de saúde mental e psiquiatria. Os participantes do estudo, em sua maioria, definem grupo conforme o abordado nos diversos referenciais teóricos sobre grupo. Alguns definem grupo como uma modalidade terapêutica; outros relacionam com reuniões multiprofissionais para a melhora na assistência prestada e outros, ainda, relacionam o conceito de grupo com a coordenação dos mesmos.

Um dos entrevistados, ao conceituar grupo, refere-se a um conjunto de pessoas reunidas com o mesmo objetivo, como pode ser observado no depoimento que se segue. ... grupo é um conjunto de pessoas que têm o mesmo objetivo (E3).

A conceituação de grupo acima mencionada difere um pouco da abordagem conceitual referida por alguns estudiosos, em que os grupos se formam quando os membros têm um objetivo em comum e não somente o mesmo objetivo $^{(3)}$.

Há distinção entre grupo propriamente dito e agrupamento. Referem que, em um agrupamento de pessoas, é compartilhado o mesmo espaço e o mesmo interesse, porém, não ocorre vínculo entre elas. Após a ocorrência de uma determinada situação, a configuração grupal pode se modificar e as pessoas se constituírem em um interativo grupo de trabalho. Os autores citam que a passagem de um agrupamento para um grupo propriamente dito consiste na transformação de interesses comuns para a de interesses em comum $^{(5)}$.

$\mathrm{O}$ que distingue um conjunto de pessoas de um grupo seria o sistema humano que se constitui em torno da interação grupal, realizando equivalência entre as expressões grupos e sistemas humanos ${ }^{(16)}$.

Outros entrevistados, no entanto, apresentam em seus depoimentos a concepção de grupo como pessoas reunidas com objetivos em comum, diferenciando-as da anteriormente citada. São pessoas que têm um objetivo em comum... que se reúnem com objetivos em comum (E8). Reunião de pessoas com o mesmo objetivo... visando o trabalho em comum... (E4).

A concepção de objetivo em comum, ou objetivo compartilhado, tem em si a ideia de ser todo aquele conjunto de pessoas capaz de se reconhecer em sua particularidade e, ao mesmo tempo, de exercer ação interativa em busca desse objetivo ${ }^{(16)}$.

O enfermeiro expressa a relação que deve existir para que um grupo se caracterize enquanto grupo. Nesse depoimento, apesar de o sujeito usar o termo "agrupam", expressa e afirma que, para existir grupo, deve haver uma relação entre as pessoas, desse modo, indo ao encontro do conceito de sistema humano de Osório ${ }^{(16)}$. Grupo para mim é quando as pessoas se agrupam com o mesmo objetivo e têm uma relação entre elas (E15).

Quanto ao conceito apontado por outro enfermeiro, sobre grupo, sua fala se aproxima da concepção de grupo citada por um estudioso do tema, quando o autor afirma que é vaga e imprecisa a definição de grupo, podendo ser o conjunto de duas ou três pessoas, como também uma família, gangue ou classe ${ }^{(3)}$. Nesse mesmo depoimento ainda foi abordada a questão do objetivo em comum. É toda atividade na qual mais que uma pessoa, mais que duas pessoas 
se reúnem para discutir ou refletir sobre temas especificos... busca algo em comum(E6).

Em outra entrevista, o enfermeiro, ao conceituar grupo, faz referência ao local onde os pacientes se encontram e às regras determinadas em um grupo, como pode ser observado a seguir. ... grupo para mim é o local onde os pacientes se encontram... onde existem algumas regras que são determinadas... (E10).

O conjunto de regras forma o enquadramento (setting) do grupo, que seria uma importante recomendação técnica para o seu estabelecimento e a sua preservação. $\mathrm{O}$ enquadramento, então, seria a soma de todos os procedimentos que organizam e possibilitam o funcionamento grupal, resultado de uma conjunção de regras como, por exemplo, o local das reuniões, os horários, a periodicidade, o plano de férias, os honorários e o número médio de participantes. Os principais elementos a serem considerados na configuração de um setting grupal são: se o grupo é aberto ou fechado, homogêneo ou heterogêneo, se sua duração é limitada ou ilimitada, o número de participantes, tipo e finalidade do grupo, duração de cada reunião, reuniões semanais ou mensais ${ }^{(2)}$.

Outra entrevista apresenta o sujeito mencionando o termo agregação de pessoas. ... acho que é uma agregração de pessoas... (E18).

Vale ressaltar a necessidade de haver interação entre os membros do grupo para que ele se caracterize como tal. Um grupo não é um mero somatório de indivíduos em que todos os seus integrantes estão reunidos em torno de um objetivo em comum $^{(3)}$.

Outros enfermeiros, ao trazerem a conceituação de grupo, referem-se à importância da coordenação e da equipe multidisciplinar para resolver questões ligadas à assistência, e ao grupo como uma modalidade terapêutica.

No depoimento seguinte, o participante faz referência à coordenação em sua conceituação. ... se não tiver coordenação já não é um grupo (E7).

Um grupo, entretanto, pode funcionar sem um coordenador propriamente dito como, por exemplo, nos grupos de autoajuda, em que pessoas com as mesmas características reúnem-se para ajuda mútua e, nesse caso, não há necessidade de um coordenador pré-estabelecido.

Os grupos de autoajuda têm como característica serem grupos de formação espontânea de pessoas que se sentem identificadas por características semelhantes entre $\mathrm{Si}^{(5)}$.

Ainda sobre os grupos de autoajuda, esses grupos organizados por uma experiência comum entre os seus membros podem ou não receber consultoria de um provedor da saúde que pode ser o enfermeiro. Porém, eles são operados pelos próprios membros ${ }^{(8)}$.

Os autores citados acima afirmam que, com exceção dos grupos de autoajuda, os outros tipos de grupos necessitam de coordenador, sendo preciso preparo e competência para a realização dessa tarefa.

O papel do coordenador de grupo é o de ordenar com alguém o pensar, o sentir e o agir de um conjunto articulado; não é comprometido emocionalmente com a situação grupal, mas não é neutro, vive e sente com o grupo, oferece apoio psicológico, acolhe necessidades ${ }^{(17)}$.
Os atributos do coordenador são parte integrante da fundamentação técnica. Além dos conhecimentos provindos do estudo, as habilidades (treino e supervisão) e as atitudes (tratamento psicanalítico) são indispensáveis para o bom funcionamento grupal ${ }^{(2)}$.

Com relação à concepção sobre a equipe multidisciplinar para resolver questões ligadas à assistência que os sujeitos fizeram para a sua conceituação de grupo, seguem os depoimentos. Um grupo é todo mundo trabalhando junto, traçando ideias para melhorar o setor... (E19). Acho que é uma reunião de várias pessoas, de várias profissões... (E12).

As reuniões de equipe são imprescindíveis para o bom andamento da assistência prestada aos usuários do serviço. É um espaço de elevado valor dentro dos serviços de psiquiatria e saúde mental, na medida em que uma equipe de trabalho tem uma tarefa grupal voltada ao tratamento adequado, que seria o objetivo, em comum, da equipe.

A tomada de decisão, após longas discussões, o pensar sobre os erros e acertos, a busca por alternativas fornecem elementos para a reflexão crítica sobre o trabalho multiprofissional e interprofissional ${ }^{(18)}$.

A equipe funcionando bem, enquanto grupo voltado para um objetivo em comum, constitui uma modalidade de grupo na vivência desses enfermeiros em sua definição sobre o que seria grupo, reportando-se à sua experiência de seu local de trabalho.

Um participante, ao conceituar grupo, refere-se a uma modalidade de tratamento. É uma modalidade de tratamento... em que pessoas reúnem-se com técnicas especificas por parte dos terapeutas para tratar de questões da doença, da vida, do dia a dia dos pacientes... (E4).

A Reforma Psiquiátrica, ocorrida no Brasil na década de 1970, apropria-se da estratégia grupal como uma modalidade de tratamento, em que a reabilitação psicossocial passou a ser a base do projeto terapêutico no tratamento das pessoas com transtorno psíquico.

O recurso grupal é estratégia importante nas ações de enfermagem, pois favorece a melhoria da qualidade de assistência ao paciente e seus familiares. Em estudo na literatura, identificou-se que as modalidades de grupo mais utilizadas por enfermeiros na área de saúde mental são: os grupos operativos, os de suporte/apoio e os grupos em sala de espera ${ }^{(19)}$.

\section{Considerações Finais}

Os resultados deste estudo possibilitaram observar que alguns dos participantes têm concepções que são condizentes com a literatura sobre grupos no que diz respeito ao conceito de grupo, enquanto outros, ao conceituarem grupos, fazem-no de forma inadequada. Por exemplo, quando se referem à questão específica da conceituação de grupo, entendem que o mesmo tem objetivos em comum, contrastando com outros enfermeiros que referiram ser grupo aquele que tem objetivos comuns. Emergiu, ainda, das falas a definição de grupo como uma agregação de pessoas.

Observa-se, ainda, em relação à conceituação de grupo, que os enfermeiros apontam para reunião de equipe multiprofissional, sendo esse um espaço importante para a 
melhoria da assistência, e que, quando todos os membros estiverem voltados para um objetivo em comum, a equipe se estabelecerá como grupo e, assim, poderá ocorrer a melhora na qualidade da assistência.

A visão da autoria deste estudo é a de que o enfermeiro que atua em Psiquiatria e Saúde Mental deve se preparar melhor para atuar em grupos, uma vez que essa atividade é muito utilizada nessa área, bem como deve haver mais investimento das instituições de ensino voltado para a formação do profissional e na instituição de saúde, para realização de grupos na assistência.

Os resultados apontam a necessidade de melhor preparo dos enfermeiros para o trabalho com grupos, principalmente em relação à coordenação dos mesmos, o que não difere dos dados encontrados na literatura a esse respeito.

Pensa-se, aqui, que a pesquisa realizada poderá contribuir para que os profissionais das instituições de saúde e ensino possam refletir sobre as questões da formação do enfermeiro na coordenação de grupos em saúde mental, para a melhoria da qualidade da assistência.

A formação do enfermeiro para atuação em grupos na área de Psiquiatria e Saúde Mental precisa ser repensada, pois, segundo alguns participantes do estudo, para se especializarem, precisaram buscar cursos e isso demanda tempo, tempo esse que nem sempre é possível devido às exigências do serviço e à política da instituição.

A temática grupo carece de melhor enfoque tanto na assistência como no ensino, para que haja aplicação apropriada desse recurso como fonte das ações de enfermagem.

\section{Referências}

1. Anzieu DY, Martim JY. La dinâmica de los grupos pequenos. Buenos Aires: Kapeluz; 1971.

2. Zimerman DE. Fundamentos teóricos. In: Zimerman DE, Osorio LC, organizadores. Como trabalhamos com grupos. Porto Alegre: Artes Médicas; 1997. p. 23-31.

3. Zimerman DE. Fundamentos básicos das grupoterapias. Porto Alegre: Artes Médicas Sul; 2000.

4. Freire M. O que é um grupo? In: Grossi EP, Brodim J, organizadores. A paixão de aprender. Rio de Janeiro: Vozes; 1996. p. 59-68.

5. Zimerman DE, Osorio LC, organizadores. Como trabalhamos com grupos. Porto Alegre: Artes Médicas; 1997.

6. Escola Politécnica de Saúde Joaquim Venâncio, organizador. Textos de apoio em saúde mental. Rio de Janeiro: Editora FIOCRUZ; 2003.

7. Corrêa AK, Souza MCBM, Saeki T. Transição para o exercício profissional em enfermagem: uma experiência em grupo operativo. Esc Anna Nery Rev Enferm. 2005;9(3):421-8.

8. Lasalle LC, Lasalle AJ. Grupos terapêuticos. In: Sturt GW, Laraia MT. Enfermagem psiquiátrica: princípio e prática. Porto Alegre: Artmed; 2001. p. 695-709.

9. Campos MA, Contel JOB. Reuniões comunitárias em um hospital-dia psiquiátrico universitário: implantação e análise preliminar da experiência. Rev ABP-APAL. 1996;18(4):111-6.

10. Bezerra JRB. Considerações sobre terapêuticas ambulatoriais em saúde mental. In: Tundis AS, Costa NR, organizadores. Cidadania e loucura: políticas de saúde mental no Brasil. Petrópolis: Vozes; 1987. p. 143-69.

11. Ribeiro V, Munari DB. Saúde mental em clientes cirúrgicos: o desenvolvimento de ações de enfermagem através do grupo de suporte/apoio. Rev Bras Enferm. 1998;51(1):147-64.

12. Godoy MTH. Análise da produção científica sobre a utilização de atividades grupais no trabalho do enfermeiro no Brasil: 1980 a 2003 [dissertação]. [Goiânia]: Universidade Federal de Goiás - Faculdade de Enfermagem; 2004. 125 p.

13. Palácios M. Ética na pesquisa em seres humanos. In: Palácios M, Martins A, Pegorano OA. Ética, ciência e saúde - desafios da bioética. Petrópolis: Vozes; 2001. p. 164-75.

14. Minayo MCS. O desafio do conhecimento: pesquisa qualitativa em saúde. São Paulo: Hucitec - Abrasco; 1996. 15. Marconi MA, Lakatos EM. Técnica de pesquisa. São Paulo: Atlas; 2002.

16. Osorio LC, organizador. Grupoterapia hoje. Artes Médicas: Porto Alegre; 1989.

17. Gayotto ML, organizador. Liderança II: aprender a coordenar grupos. Petrópolis. Rio de Janeiro: Vozes; 2003. 18. Campos MA. O trabalho em equipe multiprofissional: uma reflexão crítica. J Bras Psiquiatria. 1992;41(6):255-7. 19. Spadini LS, Souza MCM. Grupos realizados por enfermeiros na área de saúde mental. Esc Anna Nery Rev Enferm. 2006;10(1):132-8.

\section{Como citar este artigo:}

Spadini LS, Souza MCBM. Conceito de grupo na percepção de enfermeiros na área de saúde mental e psiquiatria. SMAD, Rev. Eletrônica Saúde Mental Álcool Drog. (Ed. port.). set.-dez. 2011 [acesso: Disponível em:

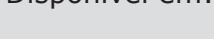

$\longrightarrow$ 